\title{
CASC15 gene polymorphisms reduce neuroblastoma risk in Chinese children
}

\author{
Jiao Zhang ${ }^{1, *}$, Zhen-Jian Zhuo ${ }^{3, *}$, Jiaxiang Wang ${ }^{1}$, Jing He ${ }^{2}$, Lin Yang ${ }^{1}$, Da Zhang ${ }^{1}$, \\ Pan Qin ${ }^{1}$ and Lizhao Yan ${ }^{1}$ \\ ${ }^{1}$ Department of Pediatric Surgery, The First Affiliated Hospital of Zhengzhou University, Zhengzhou 450052, Henan, China \\ ${ }^{2}$ Department of Pediatric Surgery, Guangzhou Institute of Pediatrics, Guangzhou Women and Children's Medical Center, \\ Guangzhou Medical University, Guangzhou 510623, Guangdong, China \\ ${ }^{3}$ School of Chinese Medicine, Faculty of Medicine, The Chinese University of Hong Kong, Hong Kong 999077, China \\ "These authors have contributed equally to this work
}

Correspondence to: Jiao Zhang, email: zhangjiaomail@126.com

Jing He, email: hejing198374@gmail.com

Keywords: neuroblastoma, CASC 15, 6p22, polymorphism, susceptibility

Received: May 25, $2017 \quad$ Accepted: July 31, $2017 \quad$ Published: August 24, 2017

Copyright: Zhang et al. This is an open-access article distributed under the terms of the Creative Commons Attribution License 3.0 (CC BY 3.0), which permits unrestricted use, distribution, and reproduction in any medium, provided the original author and source are credited.

\section{ABSTRACT}

In this case-control study, we analyzed the association between three single nucleotide polymorphisms (SNPs) in the CASC15 gene (rs6939340 A>G, rs4712653 T>C, and rs9295536 C >A) and neuroblastoma susceptibility in the Guangdong and Henan populations of China. We genotyped and analyzed 118 cases and 281 control subjects from Henan province and combined them with previously published data from the Guangdong population. In the Henan population, only the rs6939340 G>A variant homozygote AA was associated with decreased neuroblastoma risk [AA vs. GG: adjusted odds ratio $(O R)=0.47,95 \%$ confidence interval $(\mathrm{CI})=0.23-0.98$; $P=0.045]$. All three polymorphisms, individually and in combination, were associated with decreased neuroblastoma susceptibility in the Guangdong population. Moreover, subjects carrying 1-3 of these protective genotypes had lower neuroblastoma susceptibility than non-carriers (adjusted $O R=0.65,95 \% C I=0.51-0.84, P=0.0007$ ). These results show that all three genetic variants of CASC15 identified in a genomewide association study (GWAS) decrease neuroblastoma risk in two distinct Chinese populations.

\section{INTRODUCTION}

Neuroblastoma is an embryonal cancer that arises from primordial sympathetic neural precursors in infants [1]. The incidence rate of neuroblastoma is about 1 in 7000 in the USA [2] and 7.7 per million in China [3]. Neuroblastoma is the most common solid tumor in childhood and despite marked improvements in its treatment, it still accounts for $15 \%$ of the cancer-related mortality in children [4]. Neuroblastoma is characterized by diverse clinical behaviors based on which it is classified into low risk, intermediate risk and high risk groups [5]. Typically, high-risk patients have widely disseminated disease at diagnosis and poor survival rates, whereas most of the low-risk patients spontaneously regress without chemotherapy $[6,7]$. Nearly $50 \%$ of the neuroblastoma patients are diagnosed as high-risk and less than $40 \%$ of them survive despite intensive therapies $[4,8]$. Moreover, survivors face lifelong serious co-existing health issues that affect their social life [9].

Only $1 \%$ neuroblastoma cases are familial, and in most cases are associated with $A L K$ gene mutations $[10,11]$. However, the exact etiology of sporadic neuroblastoma is obscure [1]. Extrinsic risk factors that influence neuroblastoma like exposure to radiation and other environmental factors have not yet been identified. Few children develop neuroblastoma because their parents are exposed to radiation sources, wood dust 
and hydrocarbons $[12,13]$. Therefore, genetic factors combined with environmental factors can influence neuroblastoma outcomes.

The availability of the human genome sequence and high density single nucleotide polymorphism (SNP) arrays have resulted in genome-wide association studies (GWASs), which shed more light into possible genetic mechanisms of human diseases including cancers [14]. GWAS on neuroblastoma identified several susceptibility loci. SNPs in HSD17B12, DUSP12, IL31RA and DDX4 genes were enriched in the low-risk group of patients [15]. SNPs in BARD1 [16], cancer susceptibility candidate 15 (CASC15) [17], and LMO1 [18] genes were enriched in high-risk neuroblastoma patients. Also, some common variants were associated with neuroblastoma risk, but were not correlated with the disease phenotype [19]. Since its possible to miss relevant risk variants because of stringent threshold parameters in GWAS studies, alternative approaches were used to identify potential variants associated with neuroblastoma including NEFL [20] and TP53 [21]. Most GWAS-identified SNPs in neuroblastoma have been verified in other population samples [22-24]. Yet, only four studies have assessed the association of polymorphisms in CASC15 gene with neuroblastoma risk [22-25].

In our previous study, we reported that CASC15 gene polymorphisms were associated with neuroblastoma risk in Southern Chinese population [25]. In this study, we evaluate the association between CASC15 gene polymorphisms and neuroblastoma risk in two combined cohorts of neuroblastoma patients from Northern China.

\section{RESULTS}

\section{Population characteristics}

The detailed demographic characteristics of Guangdong population were reported previously [2527]. For Henan population, a summary of demographic characteristics of the 118 cases and 281 controls are shown in Table 1. The mean age at diagnosis was $46.24 \pm 29.98$ months for the cases (range 0 to 131.1 months) and $44.97 \pm$ 33.23 months for the controls (range 0.1 to 144.0 months). There were no differences in age and gender $(P=0.189$ and 0.196 , respectively) between the case and control subjects. According to the INSS criteria [28], 15 (12.82\%), 31 (26.50\%), 19 (16.24\%), 49 (41.88\%), and $3(2.56 \%)$ cases were classified into stages $\mathrm{I} \sim \mathrm{V}$, and $4 \mathrm{~s}$, respectively. Out of the 118 cases, adrenal gland, mediastinum and other regions accounted for $89(75.42 \%), 19(16.10 \%)$, and 10 $(8.47 \%)$ neuroblastomas, respectively.

\section{Association between $C A S C 15$ gene polymorphisms and neuroblastoma susceptibility}

Table 2 shows the genotype frequencies of the CASC15 gene polymorphisms in the cases and controls from Henan and Guangdong provinces, both separately and combined, and their association with neuroblastoma risk. The three polymorphisms, rs6939340 $\mathrm{G}>\mathrm{A}(P=0.970)$, rs4712653 $\mathrm{C}>\mathrm{T}(P=0.290)$ and rs9295536 $\mathrm{A}>\mathrm{C}(P=0.287)$ were in accordance with Hardy-Weinberg equilibrium (HWE) for combined subjects. In the case subjects from Henan province, we found decreased neuroblastoma risk with the rs6939340 AA genotype compared to the wildtype GG genotype (AA vs. GG: adjusted $\mathrm{OR}=0.47,95 \%$ $\mathrm{CI}=0.23-0.98, P=0.045)$. However, the two other genotypes, rs4712653 $\mathrm{C}>\mathrm{T}$ and rs9295536 $\mathrm{A}>\mathrm{C}$ were not associated with neuroblastoma risk.

For the case subjects from Guangdong, all three polymorphisms, namely, rs6939340 A allele [AA vs. GG: adjusted $\mathrm{OR}=0.55,95 \% \mathrm{CI}=0.31-0.96, P=0.036 ; \mathrm{AG} /$ AA vs. GG: adjusted $\mathrm{OR}=0.50,95 \% \mathrm{CI}=0.37-0.68$, $P<0.0001$ ], rs4712653 T (CT/TT vs. CC: adjusted $\mathrm{OR}=$ $0.57,95 \% \mathrm{CI}=0.42-0.78, P=0.0004)$ and $\mathrm{rs} 9295536 \mathrm{C}$ allele (AC/CC vs. AA: adjusted $\mathrm{OR}=0.59,95 \% \mathrm{CI}=0.43-0.80$, $P=0.0008$ ) were all associated with reduced neuroblastoma risk. When the protective genotypes were combined, the subjects with 1-3 protective genotypes were less likely to develop neuroblastoma than those not carrying the protective genotypes (adjusted $\mathrm{OR}=0.62,95 \% \mathrm{CI}=0.46-0.84, P=0.002$ ).

To strengthen the conclusion, we further analyzed the association between $C A S C 15$ gene polymorphisms and neuroblastoma risk by combining the study populations from Henan province and Guangdong province. We found that carriers of rs6939340 A allele were associated with decreased neuroblastoma risk (AA vs. GG: adjusted $\mathrm{OR}=0.50$, $95 \% \mathrm{CI}=0.32-0.78, P=0.0024 ; \mathrm{AG} / \mathrm{AA}$ vs. GG: adjusted $\mathrm{OR}=0.56,95 \% \mathrm{CI}=0.44-0.72, P<0.0001 ; \mathrm{AA}$ vs. $\mathrm{GG} / \mathrm{AG}$ : adjusted $\mathrm{OR}=0.64,95 \% \mathrm{CI}=0.42-0.99, P=0.044)$. Reduced risk was also associated with the rs4712653 T allele (CT/TT vs. $\mathrm{CC}$ : adjusted $\mathrm{OR}=0.65,95 \% \mathrm{CI}=0.51-0.89, P=0.0009)$ and rs $9295536 \mathrm{C}$ allele $(\mathrm{AC} / \mathrm{CC}$ vs. $\mathrm{AA}$ : adjusted $\mathrm{OR}=0.70$, $95 \% \mathrm{CI}=0.54-0.90, P=0.0050$ ). Moreover, subjects with 1-3 protective genotypes showed decreased neuroblastoma risk than those without the protective alleles (adjusted $\mathrm{OR}=0.65$, $95 \% \mathrm{CI}=0.51-0.84, P=0.0007$ ).

\section{False positive report probability (FPRP) analysis}

The prior probabilities ranging from 0.25 to 0.0001 were used to weigh the significant findings (Table 3). Except for rs6939340 AA vs. GG/AG and 2 protective carriers, all the significant findings remained true when we used a FPRP threshold of 0.2 as suggested by Wacholder et al. [29]. 
Table 1: Frequency distribution of selected characteristics in neuroblastoma cases and cancer-free controls

\begin{tabular}{|c|c|c|c|c|c|}
\hline \multirow[t]{2}{*}{ Variables } & \multicolumn{2}{|c|}{ Cases $(n=118)$} & \multicolumn{2}{|c|}{ Controls $(n=281)$} & \multirow[t]{2}{*}{$P^{a}$} \\
\hline & No. & $\%$ & No. & $\%$ & \\
\hline Age range, month & $0-131.1$ & & $0.1-144.0$ & & 0.189 \\
\hline Mean $\pm \mathrm{SD}$ & $46.24 \pm 29.98$ & & $44.97 \pm 33.23$ & & \\
\hline$\leq 18$ & 23 & 19.49 & 72 & 25.62 & \\
\hline$>18$ & 95 & 80.51 & 209 & 74.38 & \\
\hline Gender & & & & & 0.196 \\
\hline Female & 54 & 45.76 & 109 & 38.79 & \\
\hline Male & 64 & 54.24 & 172 & 61.21 & \\
\hline \multicolumn{6}{|l|}{ Clinical stages } \\
\hline I & 15 & 12.82 & & & \\
\hline II & 31 & 26.50 & & & \\
\hline III & 19 & 16.24 & & & \\
\hline IV & 49 & 41.88 & & & \\
\hline $4 s$ & 3 & 2.56 & & & \\
\hline \multicolumn{6}{|l|}{ Sites of origin } \\
\hline Adrenal gland & 89 & 75.42 & & & \\
\hline Mediastinum & 19 & 16.10 & & & \\
\hline Other regions & 10 & 8.47 & & & \\
\hline
\end{tabular}

${ }^{\text {aTwo-sided }} \chi^{2}$ test for distributions between neuroblastoma cases and cancer-free controls.

\section{DISCUSSION}

In the current case-control study, we investigated the potential association between CASC15 gene polymorphisms and neuroblastoma risk in two distinct populations from China. Given the relative paucity of somatic mutations in high-risk neuroblastoma, the genetic basis of sporadic neuroblastoma still needs to be elucidated. The CASC15 gene, also referred as FLJ22536 or LINC00340 gene spans $\sim 530$ kilobases on chromosome $6 \mathrm{p} 22$. It was originally identified in silico as a highly active long non-coding RNA (lncRNA) [30]. In 2008, the first GWAS was conducted in the European population to identify common DNA variations that were predisposed to sporadic neuroblastoma [17]. This GWAS consisted of the discovery stage that included 1032 cases and 2043 controls from Europe and the replication stage that included 720 cases and 2128 controls from Northern Europe. Three SNPs were identified in the CASC15 gene that associated with clinically aggressive neuroblastoma. Since then, these SNPs have been investigated in African-American and Italian populations. Latorre et al. studied 390 cases and 2500 controls in an African-American cohort and did not find any association between these 3 CASC15 gene SNPs with neuroblastoma susceptibility [22]. Capasso et al. showed that two CASC15 gene SNPs (rs6939340 A> G and rs4712653 T>C) were risk factors for neuroblastoma by evaluating 370 cases and 809 controls in an Italian cohort [23]. Mike et al. showed that decreased expression of the truncated CASC15-S isoform was associated with more advanced neuroblastoma [31]. We previously conducted a case-control study with 201 cases and 531 controls in southern China and showed that all three SNPs were protective [25]. Moreover, subjects carrying one or more protective genotypes showed lower neuroblastoma susceptibility than non-carriers. However, the findings of our previous study were limited by selection bias and small sample size. Therefore, in this study we investigated the role of the three GWAS-identified SNPs in neuroblastoma risk in the two distinct populations.

The study subjects from Henan showed significant association with neuroblastoma risk only in the rs6939340 $\mathrm{G}>\mathrm{A}$ variant and not in the $\mathrm{rs} 4712653 \mathrm{C}>\mathrm{T}$ and $\mathrm{rs} 9295536$ $\mathrm{A}>\mathrm{C}$. On the other hand, all three SNPs were associated with decreased neuroblastoma risk in the Guangdong population. Combining the two populations also resulted in similar results. The differences in the relationship between CASC15 gene polymorphisms and neuroblastoma risk among the two different populations might be ascribed to different environmental exposures as well as different genetic backgrounds. We also found that subjects with 1-3 protective genotypes showed decreased neuroblastoma 
Table 2: Association between CASC15 gene polymorphisms and neuroblastoma susceptibility

\begin{tabular}{|c|c|c|c|c|c|c|c|c|c|c|c|c|}
\hline \multirow[t]{2}{*}{ Genotype } & \multicolumn{4}{|c|}{ Henan province } & \multicolumn{4}{|c|}{ Guangdong province } & \multicolumn{4}{|c|}{ Combined } \\
\hline & $\begin{array}{c}\text { Cases } \\
(\mathrm{N}=118)\end{array}$ & $\begin{array}{l}\text { Controls } \\
(\mathrm{N}=\mathbf{2 8 1})\end{array}$ & $\begin{array}{c}\text { Adjusted } \\
\text { OR (95\% } \\
\text { CI) }{ }^{\mathrm{a}}\end{array}$ & $P^{a}$ & $\begin{array}{c}\text { Cases } \\
(\mathrm{N}=\mathbf{2 5 5})\end{array}$ & $\begin{array}{l}\text { Controls } \\
(\mathrm{N}=531)\end{array}$ & $\begin{array}{c}\text { Adjusted } \\
\text { OR (95\% } \\
\text { CI) }{ }^{a}\end{array}$ & $P^{a}$ & $\begin{array}{c}\text { Cases } \\
(\mathrm{N}=373)\end{array}$ & $\begin{array}{l}\text { Controls } \\
(\mathrm{N}=\mathbf{8 1 2})\end{array}$ & $\begin{array}{c}\text { Adjusted } \\
\text { OR (95\% } \\
\text { CI })^{a}\end{array}$ & $P^{a}$ \\
\hline \multicolumn{13}{|c|}{ rs6939340 G>A $(\mathrm{HWE}=0.970)^{\mathrm{b}}$} \\
\hline GG & $\begin{array}{c}58 \\
(49.15)\end{array}$ & $\begin{array}{c}115 \\
(40.93)\end{array}$ & 1.00 & & $\begin{array}{c}155 \\
(60.78)\end{array}$ & $232(43.69)$ & 1.00 & & $\begin{array}{c}213 \\
(57.10)\end{array}$ & $\begin{array}{c}347 \\
(42.73)\end{array}$ & 1.00 & \\
\hline AG & $\begin{array}{c}49 \\
(41.53)\end{array}$ & $\begin{array}{c}121 \\
(43.06)\end{array}$ & $\begin{array}{c}0.80(0.50- \\
1.26)\end{array}$ & 0.336 & $\begin{array}{c}81 \\
(31.76)\end{array}$ & $247(46.52)$ & $\begin{array}{c}0.49(0.36- \\
0.68)\end{array}$ & $<0.0001$ & $\begin{array}{c}130 \\
(34.85)\end{array}$ & $\begin{array}{c}368 \\
(45.32)\end{array}$ & $\begin{array}{c}0.58(0.44- \\
0.75)\end{array}$ & $<0.0001$ \\
\hline AA & $11(9.32)$ & $\begin{array}{c}45 \\
(16.01)\end{array}$ & $\begin{array}{c}0.47(0.23- \\
0.98)\end{array}$ & 0.045 & $\begin{array}{c}19 \\
(7.45)\end{array}$ & $52(9.79)$ & $\begin{array}{c}0.55(0.31- \\
0.96)\end{array}$ & 0.036 & $\begin{array}{c}30 \\
(8.04)\end{array}$ & $97(11.95)$ & $\begin{array}{c}0.50(0.32- \\
0.78)\end{array}$ & 0.0024 \\
\hline Additive & & & $\begin{array}{c}0.72(0.53- \\
0.99)\end{array}$ & 0.046 & & & $\begin{array}{c}0.61(0.48- \\
0.79)\end{array}$ & $<0.0001$ & & & $\begin{array}{c}0.65(0.54- \\
0.79)\end{array}$ & $<0.0001$ \\
\hline Dominant & $\begin{array}{c}60 \\
(50.85)\end{array}$ & $\begin{array}{c}166 \\
(59.07)\end{array}$ & $\begin{array}{c}0.71(0.46- \\
1.10)\end{array}$ & 0.121 & $\begin{array}{c}100 \\
(39.22)\end{array}$ & $299(56.31)$ & $\begin{array}{c}0.50(0.37- \\
0.68)\end{array}$ & $<0.0001$ & $\begin{array}{c}160 \\
(42.90)\end{array}$ & $\begin{array}{c}465 \\
(57.27)\end{array}$ & $\begin{array}{c}0.56(0.44- \\
0.72)\end{array}$ & $<0.0001$ \\
\hline Recessive & $\begin{array}{c}107 \\
(90.68)\end{array}$ & $\begin{array}{c}236 \\
(83.99)\end{array}$ & $\begin{array}{c}0.53(0.26- \\
1.06)\end{array}$ & 0.073 & $\begin{array}{c}236 \\
(92.55)\end{array}$ & $479(90.21)$ & $\begin{array}{c}0.74(0.43- \\
1.28)\end{array}$ & 0.286 & $\begin{array}{c}343 \\
(91.96)\end{array}$ & $\begin{array}{c}715 \\
(88.05)\end{array}$ & $\begin{array}{c}0.64(0.42- \\
0.99)\end{array}$ & 0.044 \\
\hline \multicolumn{13}{|c|}{ rs $4712653 \mathrm{C}>\mathrm{T}(\mathrm{HWE}=0.290)^{\mathrm{b}}$} \\
\hline $\mathrm{CC}$ & $\begin{array}{c}69 \\
(58.47)\end{array}$ & $\begin{array}{c}154 \\
(54.80)\end{array}$ & 1.00 & & $\begin{array}{c}171 \\
(67.06)\end{array}$ & $285(53.67)$ & 1.00 & & $\begin{array}{c}240 \\
(64.34)\end{array}$ & $\begin{array}{c}439 \\
(54.06)\end{array}$ & 1.00 & \\
\hline $\mathrm{CT}$ & $\begin{array}{c}41 \\
(34.75)\end{array}$ & $\begin{array}{c}99 \\
(35.23)\end{array}$ & $\begin{array}{c}0.93(0.58- \\
1.48)\end{array}$ & 0.754 & $\begin{array}{c}69 \\
(27.06)\end{array}$ & $209(39.36)$ & $\begin{array}{c}0.55(0.39- \\
0.77)\end{array}$ & 0.0004 & $\begin{array}{c}110 \\
(29.49)\end{array}$ & $\begin{array}{c}308 \\
(37.93)\end{array}$ & $\begin{array}{c}0.65(0.50- \\
0.86)\end{array}$ & 0.0019 \\
\hline $\mathrm{TT}$ & $8(6.78)$ & $28(9.96)$ & $\begin{array}{c}0.64(0.28- \\
1.48)\end{array}$ & 0.298 & $\begin{array}{c}15 \\
(5.88)\end{array}$ & $37(6.97)$ & $\begin{array}{c}0.68(0.36- \\
1.28)\end{array}$ & 0.230 & $\begin{array}{c}23 \\
(6.17)\end{array}$ & $65(8.00)$ & $\begin{array}{c}0.65(0.39- \\
1.07)\end{array}$ & 0.088 \\
\hline Additive & & & $\begin{array}{c}0.85(0.61- \\
1.19)\end{array}$ & 0.352 & & & $\begin{array}{c}0.67(0.52- \\
0.87)\end{array}$ & 0.0023 & & & $\begin{array}{c}0.73(0.60- \\
0.89)\end{array}$ & 0.0023 \\
\hline Dominant & $\begin{array}{c}49 \\
(41.53)\end{array}$ & $\begin{array}{c}127 \\
(45.20)\end{array}$ & $\begin{array}{c}0.87(0.56- \\
1.34)\end{array}$ & 0.515 & $\begin{array}{c}84 \\
(32.94)\end{array}$ & $246(46.33)$ & $\begin{array}{c}0.57(0.42- \\
0.78)\end{array}$ & 0.0004 & $\begin{array}{c}133 \\
(35.66)\end{array}$ & $\begin{array}{c}373 \\
(45.94)\end{array}$ & $\begin{array}{c}0.65(0.51- \\
0.84)\end{array}$ & 0.0009 \\
\hline Recessive & $\begin{array}{c}110 \\
(93.22)\end{array}$ & $\begin{array}{c}253 \\
(90.04)\end{array}$ & $\begin{array}{c}0.66(0.29- \\
1.50)\end{array}$ & 0.318 & $\begin{array}{c}240 \\
(94.12)\end{array}$ & $494(93.03)$ & $\begin{array}{c}0.84(0.45- \\
1.56)\end{array}$ & 0.581 & $\begin{array}{c}350 \\
(93.83)\end{array}$ & $\begin{array}{c}747 \\
(92.00)\end{array}$ & $\begin{array}{c}0.75(0.46- \\
1.23)\end{array}$ & 0.260 \\
\hline \multicolumn{13}{|c|}{ rs9295536 A $>C(H W E=0.287)^{b}$} \\
\hline AA & $\begin{array}{c}64 \\
(54.24)\end{array}$ & $\begin{array}{c}152 \\
(54.09)\end{array}$ & 1.00 & & $\begin{array}{c}168 \\
(65.88)\end{array}$ & $282(53.11)$ & 1.00 & & $\begin{array}{c}232 \\
(62.20)\end{array}$ & $\begin{array}{c}434 \\
(53.45)\end{array}$ & 1.00 & \\
\hline $\mathrm{AC}$ & $\begin{array}{c}42 \\
(35.59)\end{array}$ & $\begin{array}{c}99 \\
(35.23)\end{array}$ & $\begin{array}{c}1.01(0.63- \\
1.61)\end{array}$ & 0.973 & $\begin{array}{c}76 \\
(29.80)\end{array}$ & $212(39.92)$ & $\begin{array}{c}0.60(0.44- \\
0.83)\end{array}$ & 0.002 & $\begin{array}{c}118 \\
(31.64)\end{array}$ & $\begin{array}{c}311 \\
(38.30)\end{array}$ & $\begin{array}{c}0.71(0.55- \\
0.93)\end{array}$ & 0.012 \\
\hline $\mathrm{CC}$ & $\begin{array}{c}12 \\
(10.17)\end{array}$ & $\begin{array}{c}30 \\
(10.68)\end{array}$ & $\begin{array}{c}0.96(0.46- \\
1.99)\end{array}$ & 0.903 & $\begin{array}{c}11 \\
(4.31)\end{array}$ & $37(6.97)$ & $\begin{array}{c}0.50(0.25- \\
1.01)\end{array}$ & 0.054 & $\begin{array}{c}23 \\
(6.17)\end{array}$ & $67(8.25)$ & $\begin{array}{c}0.64(0.39- \\
1.06)\end{array}$ & 0.081 \\
\hline Additive & & & $\begin{array}{c}0.99(0.72- \\
1.36)\end{array}$ & 0.940 & & & $\begin{array}{c}0.65(0.50- \\
0.84)\end{array}$ & 0.001 & & & $\begin{array}{c}0.76(0.62- \\
0.93)\end{array}$ & 0.0064 \\
\hline Dominant & $\begin{array}{c}54 \\
(45.76)\end{array}$ & $\begin{array}{c}129 \\
(45.91)\end{array}$ & $\begin{array}{c}1.00(0.65- \\
1.54)\end{array}$ & 0.985 & $\begin{array}{c}87 \\
(34.12)\end{array}$ & $249(46.89)$ & $\begin{array}{c}0.59(0.43- \\
0.80)\end{array}$ & 0.0008 & $\begin{array}{c}141 \\
(37.80)\end{array}$ & $\begin{array}{c}378 \\
(46.55)\end{array}$ & $\begin{array}{c}0.70(0.54- \\
0.90)\end{array}$ & 0.0050 \\
\hline Recessive & $\begin{array}{c}106 \\
(89.83)\end{array}$ & $\begin{array}{c}251 \\
(89.32)\end{array}$ & $\begin{array}{c}0.95(0.47- \\
1.94)\end{array}$ & 0.893 & $\begin{array}{c}244 \\
(95.69)\end{array}$ & $494(93.03)$ & $\begin{array}{c}0.61(0.30- \\
1.21)\end{array}$ & 0.154 & $\begin{array}{c}350 \\
(93.83)\end{array}$ & $\begin{array}{c}745 \\
(91.75)\end{array}$ & $\begin{array}{c}0.73(0.45- \\
1.19)\end{array}$ & 0.205 \\
\hline \multicolumn{13}{|c|}{ Combined effect of protective genotypes } \\
\hline 0 & $\begin{array}{c}52 \\
(44.07)\end{array}$ & $\begin{array}{c}104 \\
(37.01)\end{array}$ & 1.00 & & $\begin{array}{c}135 \\
(52.94)\end{array}$ & $218(41.05)$ & 1.00 & & $\begin{array}{c}187 \\
(50.13)\end{array}$ & $\begin{array}{c}322 \\
(39.66)\end{array}$ & 1.00 & \\
\hline 1 & $\begin{array}{c}15 \\
(12.71)\end{array}$ & $\begin{array}{c}49 \\
(17.44)\end{array}$ & $\begin{array}{c}0.60(0.31- \\
1.17)\end{array}$ & 0.133 & $\begin{array}{c}35 \\
(13.73)\end{array}$ & $67(12.62)$ & $\begin{array}{c}0.84(0.53- \\
1.33)\end{array}$ & 0.454 & $\begin{array}{c}50 \\
(13.40)\end{array}$ & $\begin{array}{c}116 \\
(14.29)\end{array}$ & $\begin{array}{c}0.74(0.51- \\
1.08)\end{array}$ & 0.118 \\
\hline 2 & $5(4.24)$ & $11(3.91)$ & $\begin{array}{c}0.92(0.30- \\
2.79)\end{array}$ & 0.879 & $\begin{array}{c}19 \\
(7.45)\end{array}$ & $11(2.07)$ & $\begin{array}{c}2.79(1.29- \\
6.06)\end{array}$ & 0.009 & $\begin{array}{c}24 \\
(6.43)\end{array}$ & $22(2.71)$ & $\begin{array}{c}1.89(1.03- \\
3.46)\end{array}$ & 0.040 \\
\hline
\end{tabular}




\begin{tabular}{|c|c|c|c|c|c|c|c|c|c|c|c|c|}
\hline \multirow[t]{2}{*}{ Genotype } & \multicolumn{4}{|c|}{ Henan province } & \multicolumn{4}{|c|}{ Guangdong province } & \multicolumn{4}{|c|}{ Combined } \\
\hline & $\begin{array}{c}\text { Cases } \\
(\mathrm{N}=118)\end{array}$ & $\begin{array}{l}\text { Controls } \\
(\mathrm{N}=\mathbf{2 8 1})\end{array}$ & $\begin{array}{c}\text { Adjusted } \\
\text { OR }(95 \% \\
\text { CI })^{a}\end{array}$ & $P^{a}$ & $\begin{array}{c}\text { Cases } \\
(\mathrm{N}=\mathbf{2 5 5})\end{array}$ & $\begin{array}{l}\text { Controls } \\
(\mathrm{N}=531)\end{array}$ & $\begin{array}{c}\text { Adjusted } \\
\text { OR (95\% } \\
\text { CI })^{a}\end{array}$ & $P^{a}$ & $\begin{array}{c}\text { Cases } \\
(\mathrm{N}=373)\end{array}$ & $\begin{array}{l}\text { Controls } \\
(\mathrm{N}=\mathbf{8 1 2})\end{array}$ & $\begin{array}{c}\text { Adjusted } \\
\text { OR (95\% } \\
\text { CI })^{a}\end{array}$ & $P^{\mathrm{a}}$ \\
\hline 3 & $\begin{array}{c}46 \\
(38.98)\end{array}$ & $\begin{array}{c}117 \\
(41.64)\end{array}$ & $\begin{array}{c}0.78(0.49- \\
1.26)\end{array}$ & 0.315 & $\begin{array}{c}66 \\
(25.88)\end{array}$ & $235(44.26)$ & $\begin{array}{c}0.45(0.32- \\
0.64)\end{array}$ & $<0.0001$ & $\begin{array}{c}112 \\
(30.03)\end{array}$ & $\begin{array}{c}352 \\
(43.35)\end{array}$ & $\begin{array}{c}0.55(0.42- \\
0.72)\end{array}$ & $<0.0001$ \\
\hline $1-3$ & $\begin{array}{c}66 \\
(55.93)\end{array}$ & $\begin{array}{c}177 \\
(62.99)\end{array}$ & $\begin{array}{c}0.74(0.48- \\
1.15)\end{array}$ & 0.177 & $\begin{array}{c}120 \\
(47.06)\end{array}$ & $313(58.95)$ & $\begin{array}{c}0.62(0.46- \\
0.84)\end{array}$ & 0.002 & $\begin{array}{c}186 \\
(49.87)\end{array}$ & $\begin{array}{c}490 \\
(60.34)\end{array}$ & $\begin{array}{c}0.65(0.51- \\
0.84)\end{array}$ & 0.0007 \\
\hline
\end{tabular}

The results were in bold if the $95 \%$ CI excluded 1 or $P<0.05$.

a Adjusted for age and gender.

${ }^{\mathrm{b}}$ Hardy-Weinberg equilibrium (HWE) for combined subjects.

Table 3: False-positive report probability results for significant findings in combined subjects

\begin{tabular}{|c|c|c|c|c|c|c|c|c|}
\hline \multirow[t]{2}{*}{ Genotype } & \multirow{2}{*}{$\begin{array}{c}\text { Crude OR } \\
(95 \% \text { CI) }\end{array}$} & \multirow[t]{2}{*}{$P^{\text {a }}$} & \multirow{2}{*}{$\begin{array}{c}\text { Statistical } \\
\text { Power }^{b}\end{array}$} & \multicolumn{5}{|c|}{ Prior probability } \\
\hline & & & & 0.25 & 0.1 & 0.01 & 0.001 & 0.0001 \\
\hline \multicolumn{9}{|l|}{ rs6939340 G>A } \\
\hline AG vs. GG & $0.58(0.44-0.75)$ & $<0.0001$ & 0.152 & 0.001 & 0.002 & 0.024 & 0.199 & 0.714 \\
\hline AA vs. GG & $0.50(0.32-0.79)$ & 0.0025 & 0.108 & 0.065 & 0.172 & 0.695 & 0.958 & 0.996 \\
\hline AG/AA vs. GG & $0.56(0.44-0.72)$ & $<0.0001$ & 0.078 & 0.000 & 0.000 & 0.005 & 0.049 & 0.339 \\
\hline AA vs. GG/AG & $0.64(0.42-0.99)$ & 0.0451 & 0.440 & 0.235 & 0.480 & 0.910 & 0.990 & 0.999 \\
\hline \multicolumn{9}{|l|}{ rs4712653 C>T } \\
\hline TC vs. CC & $0.65(0.50-0.86)$ & 0.0019 & 0.446 & 0.013 & 0.037 & 0.297 & 0.810 & 0.977 \\
\hline TC/TT vs. CC & $0.65(0.51-0.84)$ & 0.0009 & 0.419 & 0.006 & 0.019 & 0.175 & 0.682 & 0.955 \\
\hline \multicolumn{9}{|l|}{ rs9295536 A $>C$} \\
\hline CA vs. AA & $0.71(0.54-0.93)$ & 0.0113 & 0.678 & 0.048 & 0.130 & 0.623 & 0.943 & 0.994 \\
\hline $\mathrm{CA} / \mathrm{CC}$ vs. $\mathrm{AA}$ & $0.70(0.54-0.90)$ & 0.0049 & 0.626 & 0.023 & 0.066 & 0.437 & 0.887 & 0.987 \\
\hline 2 vs. 0 & $1.88(1.02-3.44)$ & 0.0415 & 0.216 & 0.366 & 0.634 & 0.950 & 0.995 & 0.999 \\
\hline 3 vs. 0 & $0.55(0.42-0.72)$ & $<0.0001$ & 0.120 & 0.001 & 0.002 & 0.019 & 0.161 & 0.658 \\
\hline $1-3$ vs. 0 & $0.65(0.51-0.84)$ & 0.0007 & 0.420 & 0.005 & 0.015 & 0.142 & 0.625 & 0.943 \\
\hline
\end{tabular}

${ }^{a}$ Chi-square test was used to calculate the genotype frequency distributions.

b Statistical power was calculated using the number of observations in the subgroup and the OR and $P$ values in this table.

risk. Further, FPRP analysis excluded the possibility of false positive results in our study, thereby confirming our data. The discrepancy of the role of CASC15 gene polymorphisms in neuroblastoma risk might be attributed to (1) relative small sample sizes in the validation studies and (2) the variation in allele frequencies, linkage disequilibrium patterns, and environmental exposures among different ethnic populations that influence cancer risk [32].

Although this study is the first to verify the genetic role of CASC15 gene polymorphisms in two independent populations in China, it has several limitations. First, although this is the largest case-control study conducted to-date, the sample size was still not large enough. The low incidence rate of neuroblastoma in China that makes it challenging to recruit eligible subjects. Second, only three GWAS-identified SNPs in the CASC15 gene were analyzed and other potential functional SNPs were not tested. Third, the present study focused on the subjects recruited from single hospital rather than from the entire community, which may result in selection bias. Fourth, we only analyzed the genetic factors in neuroblastoma risk, whereas other environmental factors (living environment, dietary intake, and paternal exposures) were not available to conduct gene-environmental interaction analysis.

In summary, our results showed the protective role of three CASC15 gene polymorphisms in neuroblastoma 
susceptibility in two independent populations in China. Larger and multicenter-based case-control studies are warranted to further confirm the role of CASC15 gene polymorphisms in decreasing neuroblastoma risk and determine the molecular mechanism of action.

\section{MATERIALS AND METHODS}

\section{Study subjects}

This study involved two independent case-control populations. The first was derived from our previous study, which was conducted in Guangdong province [25, 26]. The second was conducted in Henan province, which included 118 cases and 281 controls. The recruitment procedure and criteria were as described previously [27, 33]. In brief, we identified 118 histologically confirmed neuroblastoma cases among Henan residents from The First Affiliated Hospital of Zhengzhou University between August 2011 and April 2017. We also recruited 281 control subjects from the same hospital during the same period. The case and control subjects were age- and gender matched. The response rate was approximately $80 \%$ for cases and $90 \%$ for controls enrolled from Henan province. All subjects or their guardians were informed about the project and gave written consent. This study was approved by the Institutional Review Board of The First Affiliated Hospital of Zhengzhou University.

\section{SNP selection and genotyping}

Three SNPs, rs6939340 G > A, rs4712653 C > $\mathrm{T}$, rs9295536 $\mathrm{A}>\mathrm{C}$ in the CASC15 gene were chosen from the previous GWAS study [17]. The genomic DNA was isolated from venous blood samples using TIANamp Blood DNA Kit (TianGen Biotech Co. Ltd., Beijing, China) according to manufacturer's instructions. Genotyping was performed by Taqman real-time PCR as published previously $[26,33]$. To ensure the accuracy of genotyping results, $10 \%$ of randomly selected samples were genotyped by DNA sequencing method. The concordance rate for the quality control samples was $100 \%$.

\section{Statistical analysis}

The goodness-of-fit $\chi^{2}$ test was used to assess if the selected SNPs deviated from Hardy-Weinberg equilibrium among controls. The two-sided chi-squared test was used to compare demographic variables and genotype frequencies of the cases and controls. ORs and their corresponding $95 \%$ CIs were computed by unconditional logistic regression analyses with or without adjusting for age and gender. The FPRP analysis was performed as described previously [29]. All reported $P$ values were two sided and a $P$ value $<0.05$ was considered statistically significant. The SAS statistical package (version 9.1; SAS Institute, Cary, NC) was used to perform all statistical analysis.

\section{ACKNOWLEDGMENTS AND FUNDING}

This study was supported by grants from National Natural Science Foundation of China (No. 81502187), Pearl River S\&T Nova Program of Guangzhou (No: 201710010086), and State Clinical Key Specialty Construction Project (Pediatric Surgery) 2013 (No: GJLCZD1301).

\section{CONFLICTS OF INTEREST}

The authors declare no competing financial interests.

\section{REFERENCES}

1. Capasso M, Diskin SJ. Genetics and genomics of neuroblastoma. Cancer Treat Res. 2010; 155:65-84.

2. Gurney JG, Ross JA, Wall DA, Bleyer WA, Severson RK, Robison LL. Infant cancer in the U.S.: histology-specific incidence and trends, 1973 to 1992. J Pediatr Hematol Oncol. 1997; 19:428-432.

3. Bao PP, Li K, Wu CX, Huang ZZ, Wang CF, Xiang YM, Peng P, Gong YM, Xiao XM, Zheng Y. Recent incidences and trends of childhood malignant solid tumors in Shanghai, 2002-2010. [Article in Chinese]. Zhonghua Er Ke Za Zhi. 2013; 51:288-294.

4. Maris JM. Recent advances in neuroblastoma. N Engl J Med. 2010; 362:2202-2211.

5. Cohn SL, Pearson AD, London WB, Monclair T, Ambros PF, Brodeur GM, Faldum A, Hero B, Iehara T, Machin D, Mosseri V, Simon T, Garaventa A, et al. The International Neuroblastoma Risk Group (INRG) classification system: an INRG Task Force report. J Clin Oncol. 2009; 27:289-297.

6. Carlsen NL. The new International Neuroblastoma Staging System: some critical notes. J Clin Oncol. 1990; 8:935-936.

7. Hero B, Simon T, Spitz R, Ernestus K, Gnekow AK, Scheel-Walter HG, Schwabe D, Schilling FH, Benz-Bohm G, Berthold F. Localized infant neuroblastomas often show spontaneous regression: results of the prospective trials NB95-S and NB97. J Clin Oncol. 2008; 26:1504-1510.

8. Matthay KK, Reynolds CP, Seeger RC, Shimada H, Adkins ES, Haas-Kogan D, Gerbing RB, London WB, Villablanca JG. Long-term results for children with high-risk neuroblastoma treated on a randomized trial of myeloablative therapy followed by 13-cis-retinoic acid: a children's oncology group study. J Clin Oncol. 2009; 27:1007-1013.

9. Kaatsch P. Epidemiology of childhood cancer. Cancer Treat Rev. 2010; 36:277-285. 
10. Mosse YP, Laudenslager M, Longo L, Cole KA, Wood A, Attiyeh EF, Laquaglia MJ, Sennett R, Lynch JE, Perri P, Laureys G, Speleman F, Kim C, et al. Identification of ALK as a major familial neuroblastoma predisposition gene. Nature. 2008; 455:930-935.

11. Osajima-Hakomori Y, Miyake I, Ohira M, Nakagawara A, Nakagawa A, Sakai R. Biological role of anaplastic lymphoma kinase in neuroblastoma. Am J Pathol. 2005; 167:213-222.

12. De Roos AJ, Olshan AF, Teschke K, Poole C, Savitz DA, Blatt J, Bondy ML, Pollock BH. Parental occupational exposures to chemicals and incidence of neuroblastoma in offspring. Am J Epidemiol. 2001; 154:106-114.

13. De Roos AJ, Teschke K, Savitz DA, Poole C, Grufferman $\mathrm{S}$, Pollock BH, Olshan AF. Parental occupational exposures to electromagnetic fields and radiation and the incidence of neuroblastoma in offspring. Epidemiology. 2001; 12:508-517.

14. Stadler ZK, Thom P, Robson ME, Weitzel JN, Kauff ND, Hurley KE, Devlin V, Gold B, Klein RJ, Offit K. Genomewide association studies of cancer. J Clin Oncol. 2010; 28:4255-4267.

15. Nguyen le B, Diskin SJ, Capasso M, Wang K, Diamond MA, Glessner J, Kim C, Attiyeh EF, Mosse YP, Cole K, Iolascon A, Devoto M, Hakonarson H, et al. Phenotype restricted genome-wide association study using a gene-centric approach identifies three low-risk neuroblastoma susceptibility Loci. PLoS Genet. 2011; 7:e1002026.

16. Capasso M, Devoto M, Hou C, Asgharzadeh S, Glessner JT, Attiyeh EF, Mosse YP, Kim C, Diskin SJ, Cole KA, Bosse K, Diamond M, Laudenslager M, et al. Common variations in BARD1 influence susceptibility to high-risk neuroblastoma. Nat Genet. 2009; 41:718-723.

17. Maris JM, Mosse YP, Bradfield JP, Hou C, Monni S, Scott RH, Asgharzadeh S, Attiyeh EF, Diskin SJ, Laudenslager M, Winter C, Cole KA, Glessner JT, et al. Chromosome 6p22 locus associated with clinically aggressive neuroblastoma. N Engl J Med. 2008; 358:2585-2593.

18. Wang K, Diskin SJ, Zhang H, Attiyeh EF, Winter C, Hou C, Schnepp RW, Diamond M, Bosse K, Mayes PA, Glessner J, Kim C, Frackelton E, et al. Integrative genomics identifies LMO1 as a neuroblastoma oncogene. Nature. 2011; 469:216-220.

19. Diskin SJ, Capasso M, Schnepp RW, Cole KA, Attiyeh EF, Hou C, Diamond M, Carpenter EL, Winter C, Lee $\mathrm{H}$, Jagannathan J, Latorre V, Iolascon A, et al. Common variation at $6 \mathrm{q} 16$ within HACE1 and LIN28B influences susceptibility to neuroblastoma. Nat Genet. 2012; 44:1126-1130.

20. Capasso M, Diskin S, Cimmino F, Acierno G, Totaro F, Petrosino G, Pezone L, Diamond M, McDaniel L, Hakonarson H, Iolascon A, Devoto M, Maris JM. Common genetic variants in NEFL influence gene expression and neuroblastoma risk. Cancer Res. 2014; 74:6913-6924.

21. Diskin SJ, Capasso M, Diamond M, Oldridge DA, Conkrite K, Bosse KR, Russell MR, Iolascon A, Hakonarson H, Devoto
M, Maris JM. Rare variants in TP53 and susceptibility to neuroblastoma. J Natl Cancer Inst. 2014; 106.

22. Latorre V, Diskin SJ, Diamond MA, Zhang H, Hakonarson H, Maris JM, Devoto M. Replication of neuroblastoma SNP association at the BARD1 locus in African-Americans. Cancer Epidemiol Biomarkers Prev. 2012; 21:658-663.

23. Capasso M, Diskin SJ, Totaro F, Longo L, De Mariano M, Russo R, Cimmino F, Hakonarson H, Tonini GP, Devoto M, Maris JM, Iolascon A. Replication of GWAS-identified neuroblastoma risk loci strengthens the role of BARD1 and affirms the cumulative effect of genetic variations on disease susceptibility. Carcinogenesis. 2013; 34:605-611.

24. Lu J, Chu P, Wang H, Jin Y, Han S, Han W, Tai J, Guo Y, Ni $\mathrm{X}$. Candidate Gene Association Analysis of Neuroblastoma in Chinese Children Strengthens the Role of LMO1. PLoS One. 2015; 10:e0127856.

25. He J, Zhang R, Zou Y, Zhu J, Yang T, Wang F, Xia H. Evaluation of GWAS-identified SNPs at 6p22 with neuroblastoma susceptibility in a Chinese population. Tumour Biol. 2016; 37:1635-1639.

26. He J, Zhong W, Zeng J, Zhu J, Zhang R, Wang F, Yang T, Zou Y, Xia H. LMO1 gene polymorphisms contribute to decreased neuroblastoma susceptibility in a Southern Chinese population. Oncotarget. 2016; 7:22770-22778. https://doi.org/10.18632/oncotarget.8178.

27. He J, Wang F, Zhu J, Zhang R, Yang T, Zou Y, Xia H. Association of potentially functional variants in the XPG gene with neuroblastoma risk in a Chinese population. J Cell Mol Med. 2016; 20:1481-1490.

28. Brodeur GM, Pritchard J, Berthold F, Carlsen NL, Castel V, Castelberry RP, De Bernardi B, Evans AE, Favrot M, Hedborg F. Revisions of the international criteria for neuroblastoma diagnosis, staging and response to treatment. Prog Clin Biol Res. 1994; 385:363-369.

29. Wacholder S, Chanock S, Garcia-Closas M, El Ghormli L, Rothman N. Assessing the probability that a positive report is false: an approach for molecular epidemiology studies. $\mathrm{J}$ Natl Cancer Inst. 2004; 96:434-442.

30. Glusman G, Qin S, El-Gewely MR, Siegel AF, Roach JC, Hood L, Smit AF. A third approach to gene prediction suggests thousands of additional human transcribed regions. PLoS Comput Biol. 2006; 2:e18.

31. Russell MR, Penikis A, Oldridge DA, Alvarez-Dominguez JR, McDaniel L, Diamond M, Padovan O, Raman P, Li Y, Wei JS, Zhang S, Gnanchandran J, Seeger R, et al. CASC15-S Is a Tumor Suppressor lncRNA at the 6p22 Neuroblastoma Susceptibility Locus. Cancer Res. 2015; 75:3155-3166.

32. Kraft P, Zeggini E, Ioannidis JP. Replication in genomewide association studies. Stat Sci. 2009; 24:561-573.

33. He J, Wang MY, Qiu LX, Zhu ML, Shi TY, Zhou XY, Sun MH, Yang YJ, Wang JC, Jin L, Wang YN, Li J, Yu HP, et al. Genetic variations of mTORC1 genes and risk of gastric cancer in an Eastern Chinese population. Mol Carcinog. 2013; 52 :E70-79. 